Kragujevac Journal of Mathematics

Volume 38(2) (2014), Pages 303-314.

\title{
ON THE WEIGHTED OSTROWSKI TYPE INEQUALITIES FOR DOUBLE INTEGRALS
}

\author{
MEHMET ZEKI SARIKAYA ${ }^{1}$, HATICE YALDIZ ${ }^{2}$, AND SAMET ERDEN $^{3}$
}

\begin{abstract}
In this paper, we obtain weighted Ostrowski type inequalities for func-
\end{abstract} tion whose second order partial derivatives are bounded.

\section{INTRODUCTION}

In 1938, the classical integral inequality established by Ostrowski [8] as follows.

Theorem 1.1. Let $f:[a, b] \rightarrow \mathbb{R}$ be a differentiable mapping on $(a, b)$ whose derivative $f^{\prime}:(a, b) \rightarrow \mathbb{R}$ is bounded on $(a, b)$, i.e., $\left\|f^{\prime}\right\|_{\infty}=\sup _{t \in(a, b)}\left|f^{\prime}(t)\right|<\infty$. Then, the inequality holds

$$
\left|f(x)-\frac{1}{b-a} \int_{a}^{b} f(t) d t\right| \leq\left[\frac{1}{4}+\frac{\left(x-\frac{a+b}{2}\right)^{2}}{(b-a)^{2}}\right](b-a)\left\|f^{\prime}\right\|_{\infty},
$$

for all $x \in[a, b]$. The constant $\frac{1}{4}$ is the best possible.

Inequality (1.1) has wide applications in numerical analysis and in the theory of some special means; estimating error bounds for some special means, some mid-point, trapezoid and Simpson rules and quadrature rules, etc. Hence inequality (1.1) has attracted considerable attention and interest from mathematicans and researchers. Due to this, over the years, the interested reader is also refered to ([1]-[7],[9]-[20]) for integral inequalities in several independent variables. In addition, the current approach of obtaining the bounds, for a particular quadrature rule, have depended on the use of Peano kernel. The general approach in the past has involved the assumption of bounded derivatives of degree greater than one.

Key words and phrases. Ostrowski's inequality, Montgomery's identities, Double integrals. 2010 Mathematics Subject Classification. Primary: 26D07. Secondary: 26D15.

Received: July 2, 2014

Accepted: October 9, 2014. 
If $f:[a, b] \rightarrow \mathbb{R}$ is differentiable on $[a, b]$ with the first derivative $f^{\prime}$ integrable on $[a, b]$, then Montgomery identity holds:

$$
f(x)=\frac{1}{b-a} \int_{a}^{b} f(t) d t+\int_{a}^{b} P(x, t) f^{\prime}(t) d t,
$$

where $P(x, t)$ is the Peano kernel defined by

$$
P(x, t):= \begin{cases}\frac{t-a}{b-a}, & a \leq t<x \\ \frac{t-b}{b-a}, & x \leq t \leq b .\end{cases}
$$

In [3] and [5], the authors obtain two identities which generalize (1.2) for functions of two variables. In fact, for $f:[a, b] \times[c, d] \rightarrow \mathbb{R}$ such that the partial derivative $\frac{\partial f(t, s)}{\partial t}, \frac{\partial f(t, s)}{\partial s}$, and $\frac{\partial^{2} f(t, s)}{\partial t \partial s}$ all exist and are continuous on $[a, b] \times[c, d]$, for all $(x, y) \in$ $[a, b] \times[c, d]$, they obtain:

$$
\begin{aligned}
(d-c)(b-a) f(x, y)= & -\int_{a}^{b} \int_{c}^{d} f(t, s) d s d t+(d-c) \int_{a}^{b} f(t, y) d t \\
& +(b-a) \int_{c}^{d} f(x, s) d s+\int_{a}^{b} \int_{c}^{d} p(x, t) p(y, s) \frac{\partial^{2} f(t, s)}{\partial t \partial s} d s d t
\end{aligned}
$$

and

$$
\begin{aligned}
(d-c)(b-a) f(x, y)= & \int_{a}^{b} \int_{c}^{d} f(t, s) d s d t+\int_{a}^{b} \int_{c}^{d} p(x, t) \frac{\partial f(t, s)}{\partial t} d s d t \\
& +\int_{a}^{b} \int_{c}^{d} q(y, s) \frac{\partial f(t, s)}{\partial s} d s d t+\int_{a}^{b} \int_{c}^{d} p(x, t) p(y, s) \frac{\partial^{2} f(t, s)}{\partial t \partial s} d s d t
\end{aligned}
$$

where

and

$$
p(x, t)= \begin{cases}t-a, & a \leq t<x \\ t-b, & x \leq t \leq b\end{cases}
$$

$$
q(y, s)= \begin{cases}s-c, & c \leq s<y \\ s-d, & y \leq s \leq d\end{cases}
$$

Definition 1.1. Let $w:(a, b) \rightarrow[0, \infty)$ be an integrable function, i. e. $\int_{a}^{b} w(t) d t<\infty$, then define $m_{i}(a, b)=\int_{a}^{b} t^{i} w(t) d t, i=0,1, \ldots$ as the $i^{t h}$ moment of $w$. 
Definition 1.2. Define the mean of the interval $[a, b]$ with respect to the density $w$ as

$$
\mu(a, b)=\frac{m_{1}(a, b)}{m_{0}(a, b)}
$$

and the variance by

$$
\sigma^{2}(a, b)=\frac{m_{2}(a, b)}{m_{0}(a, b)}-\mu^{2}(a, b)
$$

The main aim of this paper is to establish weighted Ostrowski type inequalities for function whose second order partial derivatives are bounded.

\section{Main Results}

In order to prove main results we need the following lemma:

Lemma 2.1. Let $f:[a, b] \times[c, d] \rightarrow \mathbb{R}$ be an absolutely continuous function such that the partial derivative $\frac{\partial^{2} f(t, s)}{\partial t \partial s}$ exists for all $(t, s) \in[a, b] \times[c, d]$. Then, we have

$$
\begin{aligned}
& m_{0}(a, b) m_{0}(c, d)(x-\mu(a, b))(y-\mu(c, d)) f(x, y) \\
& -m_{0}(c, d)(y-\mu(c, d))\left[\int_{a}^{x}\left(\int_{a}^{t} w(u) d u\right) f(t, y) d t+\int_{x}^{b}\left(\int_{b}^{t} w(u) d u\right) f(t, y) d t\right] \\
& -m_{0}(a, b)(x-\mu(a, b))\left[\int_{c}^{y}\left(\int_{c}^{s} w(v) d v\right) f(x, s) d s+\int_{y}^{s}\left(\int_{d}^{s} w(v) d v\right) f(x, s) d s\right] \\
& +m_{0}(a, b) m_{0}(c, d) \int_{a}^{b} \int_{c}^{d} f(t, s) d s d t=\int_{a}^{b} \int_{c}^{d} P(x, t) Q(y, s) f_{t s}(t, s) d s d t .
\end{aligned}
$$

Proof. We define the following functions:

$$
P(x, t)= \begin{cases}\int_{a}^{t}(t-u) w(u) d u, & a \leq t<x \\ \int_{b}^{t}(t-u) w(u) d u, & x \leq t \leq b\end{cases}
$$

and

$$
Q(y, s)= \begin{cases}\int_{c}^{s}(s-v) w(v) d v, & c \leq s<y \\ \int_{d}^{s}(s-v) w(v) d v, & y \leq s \leq d\end{cases}
$$


for all $(x, y) \in[a, b] \times[c, d]$. Thus, by definitions of $P(x, t)$ and $Q(y, s)$, we have

$$
\begin{aligned}
& \int_{a}^{b} \int_{c}^{d} P(x, t) Q(y, s) f_{t s}(t, s) d s d t \\
& =\int_{a}^{x} \int_{c}^{y}\left(\int_{a}^{t}(t-u) w(u) d u\right)\left(\int_{c}^{s}(s-v) w(v) d v\right) f_{t s}(t, s) d s d t \\
& +\int_{a}^{x} \int_{y}^{d}\left(\int_{a}^{t}(t-u) w(u) d u\right)\left(\int_{d}^{s}(s-v) w(v) d v\right) f_{t s}(t, s) d s d t \\
& +\int_{x}^{b} \int_{c}^{y}\left(\int_{b}^{t}(t-u) w(u) d u\right)\left(\int_{c}^{s}(s-v) w(v) d v\right) f_{t s}(t, s) d s d t \\
& +\int_{x}^{b} \int_{y}^{t}\left(\int_{b}^{t}(t-u) w(u) d u\right)\left(\int_{d}^{s}(s-v) w(v) d v\right) f_{t s}(t, s) d s d t \\
& =I_{1}+I_{2}+I_{3}+I_{4} .
\end{aligned}
$$

Integrating by parts, we can state:

$$
\begin{aligned}
I_{1}= & \int_{a}^{x}\left(\int_{a}^{t}(t-u) w(u) d u\right) \\
& \times\left\{\left.\left(\int_{c}^{s}(s-v) w(v) d v\right) f_{t}(t, s)\right|_{s=c} ^{y}-\int_{c}^{y}\left(\int_{c}^{s} w(v) d v\right) f_{t}(t, s) d s\right\} d t \\
= & \int_{a}^{x}\left(\int_{a}^{t}(t-u) w(u) d u\right) \\
& \times\left\{\left(\int_{c}^{y}(y-v) w(v) d v\right) f_{t}(t, y)-\int_{c}^{y}\left(\int_{c}^{s} w(v) d v\right) f_{t}(t, s) d s\right\} d t \\
= & \left.\int_{c}^{y}(y-v) w(v) d v\right) \int_{a}^{x}\left(\int_{a}^{t}(t-u) w(u) d u\right) f_{t}(t, y) d t \\
& -\int_{c}^{y}\left(\int_{c}^{s} w(v) d v\right)\left[\int_{a}^{x}\left(\int_{a}^{t}(t-u) w(u) d u\right) f_{t}(t, s) d t\right] d s
\end{aligned}
$$




$$
\begin{aligned}
= & \left(\int_{c}^{y}(y-v) w(v) d v\right)\left[\left.\left(\int_{a}^{t}(t-u) w(u) d u\right) f(t, y)\right|_{t=a} ^{x}\right. \\
& \left.-\int_{a}^{x}\left(\int_{a}^{t} w(u) d u\right) f(t, y) d t\right] \\
& \left.-\int_{c}^{y}\left(\int_{c}^{s} w(v) d v\right)\right]\left.\left(\int_{a}^{t}(t-u) w(u) d u\right) f(t, s)\right|_{t=a} ^{x} \\
& \left.-\int_{a}^{x}\left(\int_{a}^{t} w(u) d u\right) f(t, s) d t\right] d s \\
= & \left(\int_{a}^{x}(x-u) w(u) d u\right)\left(\int_{c}^{y}(y-v) w(v) d v\right) f(x, y) \\
& \left.-\int_{c}^{y}(y-v) w(v) d v\right)\left(\int_{a}^{x}\left(\int_{a}^{t} w(u) d u\right) f(t, y) d t\right) \\
& -\left[\int_{a}^{y}\left(\int_{c}^{s}\left(\int_{c}^{x} w(v) d v\right)\left(\int_{a}^{x}(x-u) w(u) d u\right) f(x, s) d s\right]\right. \\
& \left.+\int_{c}^{s} w(u) d u\right)(t, s) d s d t
\end{aligned}
$$

with similar methods

$$
\begin{aligned}
I_{2}= & \left(\int_{a}^{x}(x-u) w(u) d u\right)\left(\int_{y}^{d}(y-v) w(v) d v\right) f(x, y) \\
& -\left(\int_{y}^{d}(y-v) w(v) d v\right)\left(\int_{a}^{x}\left(\int_{a}^{t} w(u) d u\right) f(t, y) d t\right) \\
& -\left[\int_{y}^{d}\left(\int_{c}^{s} w(v) d v\right)\left(\int_{a}^{x}(x-u) w(u) d u\right) f(x, s) d s\right] \\
& +\int_{a}^{x} \int_{y}^{d}\left(\int_{a}^{t} w(u) d u\right)\left(\int_{d}^{s} w(v) d v\right) f(t, s) d s d t,
\end{aligned}
$$




$$
\begin{aligned}
& I_{3}=\left(\int_{x}^{b}(x-u) w(u) d u\right)\left(\int_{c}^{y}(y-v) w(v) d v\right) f(x, y) \\
& -\left(\int_{c}^{y}(y-v) w(v) d v\right)\left(\int_{x}^{b}\left(\int_{b}^{t} w(u) d u\right) f(t, y) d t\right) \\
& -\left[\int_{c}^{y}\left(\int_{c}^{s} w(v) d v\right)\left(\int_{x}^{b}(x-u) w(u) d u\right) f(x, s) d s\right] \\
& +\int_{x}^{b} \int_{c}^{y}\left(\int_{b}^{t} w(u) d u\right)\left(\int_{c}^{s} w(v) d v\right) f(t, s) d s d t, \\
& I_{4}=\left(\int_{x}^{b}(x-u) w(u) d u\right)\left(\int_{y}^{d}(y-v) w(v) d v\right) f(x, y) \\
& -\left(\int_{y}^{d}(y-v) w(v) d v\right)\left(\int_{x}^{b}\left(\int_{b}^{t} w(u) d u\right) f(t, y) d t\right) \\
& -\left[\int_{y}^{d}\left(\int_{d}^{s} w(v) d v\right)\left(\int_{x}^{b}(x-u) w(u) d u\right) f(x, s) d s\right] \\
& +\int_{x}^{b} \int_{y}^{d}\left(\int_{b}^{t} w(u) d u\right)\left(\int_{d}^{s} w(v) d v\right) f(t, s) d s d t .
\end{aligned}
$$

Adding $I_{1}, I_{2}, I_{3}$ and $I_{4}$ and rewriting, we easily deduce:

$$
\begin{aligned}
& \left(\int_{a}^{b}(x-u) w(u) d u\right)\left(\int_{c}^{d}(y-v) w(v) d v\right) f(x, y) \\
& -\left(\int_{c}^{d}(y-v) w(v) d v\right)\left[\int_{a}^{x}\left(\int_{a}^{t} w(u) d u\right) f(t, y) d t+\int_{x}^{b}\left(\int_{b}^{t} w(u) d u\right) f(t, y) d t\right] \\
& -\left(\int_{a}^{b}(x-u) w(u) d u\right)\left[\int_{c}^{y}\left(\int_{c}^{s} w(v) d v\right) f(x, s) d s+\int_{y}^{s}\left(\int_{d}^{s} w(v) d v\right) f(x, s) d s\right]
\end{aligned}
$$




$$
\begin{aligned}
& +\int_{a}^{b} \int_{c}^{d}\left(\int_{a}^{b} w(u) d u\right)\left(\int_{c}^{d} w(v) d v\right) f(t, s) d s d t \\
& =\int_{a}^{b} \int_{c}^{d} P(x, t) Q(y, s) f_{t s}(t, s) d s d t
\end{aligned}
$$

which completes the proof.

Theorem 2.1. Let $f:[a, b] \times[c, d] \rightarrow \mathbb{R}$ be an absolutely continuous function such that the partial derivative $\frac{\partial^{2} f(t, s)}{\partial t \partial s}$ exists and is bounded, i.e.,

$$
\left\|\frac{\partial^{2} f(t, s)}{\partial t \partial s}\right\|_{\infty}=\sup _{(t, s) \in(a, b) \times(c, d)}\left|\frac{\partial^{2} f(t, s)}{\partial t \partial s}\right|<\infty
$$

for all $(t, s) \in[a, b] \times[c, d]$. Then, we have

$$
\begin{aligned}
|F(x, y)| \leq & \frac{m_{0}(a, b) m_{0}(c, d)}{4}\left[(x-\mu(a, b))^{2}+\sigma^{2}(a, b)\right] \times \\
& \times\left[(y-\mu(c, d))^{2}+\sigma^{2}(c, d)\right]\left\|\frac{\partial^{2} f(t, s)}{\partial t \partial s}\right\|_{\infty} \\
\leq & \frac{m_{0}(a, b) m_{0}(c, d)}{4}\left(\left|x-\frac{a+b}{2}\right|+\frac{b-a}{2}\right)^{2} \times \\
& \times\left(\left|y-\frac{c+d}{2}\right|+\frac{d-c}{2}\right)^{2}\left\|\frac{\partial^{2} f(t, s)}{\partial t \partial s}\right\|_{\infty}
\end{aligned}
$$

where

$$
\begin{aligned}
& F(x, y)=m_{0}(a, b) m_{0}(c, d)(x-\mu(a, b))(y-\mu(c, d)) f(x, y) \\
& -m_{0}(c, d)(y-\mu(c, d))\left[\int_{a}^{x}\left(\int_{a}^{t} w(u) d u\right) f(t, y) d t+\int_{x}^{b}\left(\int_{b}^{t} w(u) d u\right) f(t, y) d t\right] \\
& -m_{0}(a, b)(x-\mu(a, b))\left[\int_{c}^{y}\left(\int_{c}^{s} w(v) d v\right) f(x, s) d s+\int_{y}^{d}\left(\int_{d}^{s} w(v) d v\right) f(x, s) d s\right] \\
& +m_{0}(a, b) m_{0}(c, d) \int_{a}^{b} \int_{c}^{d} f(t, s) d s d t .
\end{aligned}
$$


Proof. From Lemma 2.1 and using the properties of modulus, we obtain that

$$
\begin{aligned}
& \mid m_{0}(a, b) m_{0}(c, d)(x-\mu(a, b))(y-\mu(c, d)) f(x, y) \\
& -m_{0}(c, d)(y-\mu(c, d))\left[\int_{a}^{x}\left(\int_{a}^{t} w(u) d u\right) f(t, y) d t+\int_{x}^{b}\left(\int_{b}^{t} w(u) d u\right) f(t, y) d t\right] \\
& -m_{0}(a, b)(x-\mu(a, b))\left[\int_{c}^{y}\left(\int_{c}^{s} w(v) d v\right) f(x, s) d s+\int_{y}^{d}\left(\int_{d}^{s} w(v) d v\right) f(x, s) d s\right] \\
& +m_{0}(a, b) m_{0}(c, d) \int_{a}^{b} \int_{c}^{d} f(t, s) d s d t \mid \\
& \leq \int_{a}^{b} \int_{c}^{d}|P(x, t)||Q(y, s)| \frac{\partial^{2} f(t, s)}{\partial t \partial s} \mid d s d t
\end{aligned}
$$

$$
\leq\left\|\frac{\partial^{2} f(t, s)}{\partial t \partial s}\right\|_{\infty} \int_{a}^{b} \int_{c}^{d}|P(x, t)||Q(y, s)| d s d t .
$$

Now, using the change of order of integration we get

$$
\begin{aligned}
\int_{a}^{b}|P(x, t)| d t & =\int_{a}^{x} \int_{a}^{t}(t-u) w(u) d u d t+\int_{x}^{b} \int_{b}^{t}(t-u) w(u) d u d t \\
& =\frac{1}{2} \int_{a}^{b}(x-t)^{2} w(t) d t \\
& =\frac{1}{2}\left[x^{2} m_{0}(a, b)-2 x m_{1}(a, b)+m_{2}(a, b)\right] \\
& =\frac{m_{0}(a, b)}{2}\left[(x-\mu(a, b))^{2}+\sigma^{2}(a, b)\right]
\end{aligned}
$$

and similarly,

$$
\begin{aligned}
\int_{c}^{d}|Q(y, s)| d s & =\int_{c}^{y}\left(\int_{c}^{s}(s-v) w(v) d v\right) d s+\int_{y}^{d}\left(\int_{d}^{s}(s-v) w(v) d v\right) d s \\
& =\frac{1}{2} \int_{c}^{d}(y-s)^{2} w(s) d s
\end{aligned}
$$




$$
=\frac{m_{0}(c, d)}{2}\left[(y-\mu(c, d))^{2}+\sigma^{2}(c, d)\right]
$$

Thus, using (2.3) and (2.4) in (2.2), we obtain the first inequality of (2.1). To obtain the second inequality of (2.1) note that

$$
\begin{aligned}
\int_{a}^{b}(x-t)^{2} w(t) d t & \leq \sup _{t \in[a, b]}(x-t)^{2} \cdot m_{0}(a, b) \\
& =m_{0}(a, b) \max \left\{(x-a)^{2},(x-b)^{2}\right\} \\
& =m_{0}(a, b) \frac{1}{2}\left((x-a)^{2}+(x-b)^{2}+\left|(x-a)^{2}-(x-b)^{2}\right|\right) \\
& =m_{0}(a, b)\left(\left|x-\frac{a+b}{2}\right|+\frac{b-a}{2}\right)^{2}
\end{aligned}
$$

and similarly $\int_{c}^{d}(y-s)^{2} w(s) d s \leq m_{0}(c, d)\left(\left|y-\frac{c+d}{2}\right|+\frac{d-c}{2}\right)^{2}$ which upon substitution into (2.2) the proof is completed.

Remark 2.1. If we choose $(x, y)=\left(\frac{\mu(a, b)}{2}, \frac{\mu(c, d)}{2}\right)$ in Theorem 2.1, then the inequalities (2.1) reduce the following inequalities

$$
\begin{aligned}
& \mid \frac{m_{1}(a, b) m_{1}(c, d)}{4} f\left(\frac{\mu(a, b)}{2}, \frac{\mu(c, d)}{2}\right)-\left[\int_{a}^{\frac{\mu(a, b)}{2}}\left(\int_{a}^{t} w(u) d u\right) f\left(t, \frac{\mu(c, d)}{2}\right) d t\right. \\
& \left.\quad+\int_{\frac{\mu(a, b)}{2}}^{b}\left(\int_{b}^{t} w(u) d u\right) f\left(t, \frac{\mu(c, d)}{2}\right) d t\right] \frac{m_{1}(c, d)}{2} \\
& \quad-\frac{m_{1}(a, b)}{2}\left[\int_{c}^{\frac{\mu(c, d)}{2}}\left(\int_{c}^{s} w(v) d v\right) f\left(\frac{\mu(a, b)}{2}, s\right) d s\right. \\
& \left.\quad+\int_{\frac{\mu(c, d)}{2}}^{d}\left(\int_{d}^{s} w(v) d v\right) f\left(\frac{\mu(a, b)}{2}, s\right) d s\right]+m_{0}(a, b) m_{0}(c, d) \int_{a}^{b} \int_{c}^{d} f(t, s) d s d t \mid
\end{aligned}
$$




$$
\begin{aligned}
\leq & \frac{m_{0}(a, b) m_{0}(c, d)}{4}\left[\frac{\mu^{2}(a, b)}{4}+\sigma^{2}(a, b)\right]\left[\frac{\mu^{2}(c, d)}{4}+\sigma^{2}(c, d)\right]\left\|\frac{\partial^{2} f(t, s)}{\partial t \partial s}\right\|_{\infty} \\
(2.5) \leq & \frac{m_{0}(a, b) m_{0}(c, d)}{4}\left(\left|\frac{\mu(a, b)}{2}-\frac{a+b}{2}\right|+\frac{b-a}{2}\right)^{2} \times \\
& \times\left(\left|\frac{\mu(c, d)}{2}-\frac{c+d}{2}\right|+\frac{d-c}{2}\right)^{2}\left\|\frac{\partial^{2} f(t, s)}{\partial t \partial s}\right\|_{\infty} .
\end{aligned}
$$

Substituting $w(u)=1$ in (1.5) and (1.6) it follows that $m_{0}(a, b)=b-a$, $m_{1}(a, b)=\int_{a}^{b} u d u=\frac{b^{2}-a^{2}}{2}$

$$
\mu(a, b)=\frac{\int_{a}^{b} u d u}{\int_{a}^{b} d u}=\frac{a+b}{2} \quad \text { and } \quad \sigma^{2}(\mathrm{a}, \mathrm{b})=\frac{\int_{\mathrm{a}}^{\mathrm{b}} \mathrm{u}^{2} \mathrm{du}}{\int_{\mathrm{a}}^{\mathrm{b}} \mathrm{du}}=\frac{(\mathrm{b}-\mathrm{a})^{2}}{12}
$$

Substituting into (2.5) gives

$$
\begin{aligned}
& \mid \frac{\left(b^{2}-a^{2}\right)\left(d^{2}-c^{2}\right)}{16} f\left(\frac{a+b}{4}, \frac{c+d}{4}\right)-\frac{d^{2}-c^{2}}{4}\left[\int_{a}^{\frac{a+b}{4}}(t-a) f\left(t, \frac{c+d}{4}\right) d t\right. \\
& \left.\quad+\int_{\frac{a+b}{4}}^{b}(t-b) f\left(t, \frac{c+d}{4}\right) d t\right]-\frac{b^{2}-a^{2}}{4}\left[\int_{c}^{\frac{c+d}{4}}(s-c) f\left(\frac{a+b}{4}, s\right) d s\right. \\
& \left.\quad+\int_{\frac{c+d}{4}}^{d}(s-d) f\left(\frac{a+b}{4}, s\right) d s\right]+(b-a)(d-c) \int_{a}^{b} \int_{c}^{d} f(t, s) d s d t \mid \\
& \leq \frac{(b-a)(d-c)}{4}\left[\frac{(a+b)^{2}}{16}+\frac{(b-a)^{2}}{12}\right]\left[\frac{(c+d)^{2}}{16}+\frac{(d-c)^{2}}{12}\right]\left\|\frac{\partial^{2} f(t, s)}{\partial t \partial s}\right\|_{\infty} \\
& \leq \frac{(b-a)^{2}(d-c)^{2}}{32}\left\|\frac{\partial^{2} f(t, s)}{\partial t \partial s}\right\|_{\infty} \cdot
\end{aligned}
$$


Substituting $w(u)=\ln \left(\frac{1}{u}\right), a=c=0, b=d=1$ in (1.5) and (1.6) it follows that $m_{0}(0,1)=1, m_{1}(0,1)=\int_{0}^{1} u \ln \left(\frac{1}{u}\right) d u=\frac{1}{4}$

$$
\mu(0,1)=\frac{\int_{0}^{1} u \ln \left(\frac{1}{u}\right) d u}{\int_{0}^{1} \ln \left(\frac{1}{u}\right) d u}=\frac{1}{4} \quad \text { and } \quad \sigma^{2}(0,1)=\frac{\int_{0}^{1} u^{2} \ln \left(\frac{1}{u}\right) d u}{\int_{0}^{1} \ln \left(\frac{1}{u}\right) d u}-\left(\frac{1}{4}\right)^{2}=\frac{7}{144} .
$$

Substituting into (2.5) gives

$$
\begin{aligned}
& \frac{1}{4^{3}} f\left(\frac{1}{8}, \frac{1}{8}\right)+\int_{0}^{1} \int_{0}^{1} f(t, s) d s d t \\
& -\frac{1}{8}\left[\int_{0}^{\frac{1}{8}}\left(t \ln \left(\frac{1}{t}\right)+t\right) f\left(t, \frac{1}{8}\right) d t+\int_{\frac{1}{8}}^{1}\left(t \ln \left(\frac{1}{t}\right)+t-1\right) f\left(t, \frac{1}{8}\right) d t\right] \\
& -\frac{1}{8}\left[\int_{0}^{\frac{1}{8}}\left(s \ln \left(\frac{1}{s}\right)+s\right) f\left(\frac{1}{8}, s\right) d s+\int_{\frac{1}{8}}^{1}\left(s \ln \left(\frac{1}{s}\right)+s-1\right) f\left(\frac{1}{8}, s\right) d s\right] \\
& \leq \frac{1}{4^{5}}\left[1+\frac{1}{36}\right]^{2}\left\|\frac{\partial^{2} f(t, s)}{\partial t \partial s}\right\|_{\infty} \leq \frac{81}{4^{5}}\left\|\frac{\partial^{2} f(t, s)}{\partial t \partial s}\right\|_{\infty} .
\end{aligned}
$$

\section{REFERENCES}

[1] F. Ahmad, N. S. Barnett and S. S. Dragomir, New Weighted Ostrowski and Cebysev Type Inequalities, Nonlinear Analysis: Theory, Methods \& Appl., 71(12) (2009), 1408-1412.

[2] F. Ahmad, A. Rafiq, N. A. Mir, Weighted Ostrowski type inequality for twice differentiable mappings, Global Journal of Research in Pure and Applied Math., 2(2) (2006), 147-154.

[3] N. S. Barnett and S. S. Dragomir, An Ostrowski type inequality for double integrals and applications for cubature formulae, Soochow J. Math., 27(1) (2001), 109-114.

[4] N. S. Barnett, S. S. Dragomir and C. E. M. Pearce, A Quasi-trapezoid inequality for double integrals, ANZIAM J., 44 (2003), 355-364.

[5] S. S. Dragomir, P. Cerone, N. S. Barnett and J. Roumeliotis, An inequlity of the Ostrowski type for double integrals and applications for cubature formulae, Tamsui Oxf. J. Math., 16(1), (2000), 1-16.

[6] S. S. Dragomir, N. S. Barnett and P. Cerone, An n-dimensional version of Ostrowski's inequality for mappings of Hölder type, RGMIA Res. Pep. Coll., 2(2), (1999), 169-180.

[7] S. Hussain, M. A. Latif and M. Alomari, Generalized duble-integral Ostrowski type inequalities on time scales, Appl. Math. Letters, 24 (2011), 1461-1467.

[8] A. M. Ostrowski, Über die absolutabweichung einer differentiebaren funktion von ihrem integralmitelwert, Comment. Math. Helv. 10 (1938), 226-227.

[9] B. G. Pachpatte, On an inequality of Ostrowski type in three independent variables, J. Math. Anal. Appl., 249 (2000), 583-591. 
[10] B. G. Pachpatte, On a new Ostrowski type inequality in two independent variables, Tamkang J. Math., 32(1), (2001), 45-49

[11] B. G. Pachpatte, A new Ostrowski type inequality for double integrals, Soochow J. Math., 32(2), (2006), 317-322.

[12] J. Pecaric and A. Vukelic, Montgomery's identities for function of two variables, J. Math. Anal. Appl. 332 (2007), 617-630.

[13] A. Qayyum, A weighted Ostrowski-Grüss type inequality and applications, Proceeding of the World Cong. on Engineering, 2 (2009), 1-9.

[14] A. Rafiq and F. Ahmad, Another weighted Ostrowski-Grüss type inequality for twice differentiable mappings, Kragujevac Journal of Mathematics, 31 (2008), 43-51.

[15] U. M. Ozkan and H. Yıldırım, Ostrowski type inequality for double integrals on time scales, Acta Appl. Math., 110 (2010) 283-288.

[16] U. M. Ozkan and H. Yıldırım, The Grüss type inequalities for double integrals on time scales, Comp.Math. with Appl., 57(3), (2009), 436-444.

[17] M. Z. Sarikaya, On the Ostrowski type integral inequality, Acta Math. Univ. Comenianae, Vol. LXXIX(1), (2010), 129-134.

[18] M. Z. Sarikaya On the Ostrowski type integral inequality for double integrals, Demonstratio Mathematica, Vol. XLV No 3 (2012)

[19] M. Z. Sarikaya and H. Ogunmez, On the weighted Ostrowski type integral inequality for double integrals, The Arabian Journal for Science and Engineering (AJSE)-Mathematics, (2011) 36:1153-1160.

[20] N. Ujević, Some double integral inequalities and applications, Appl. Math. E-Notes, 7 (2007), 93-101.

1,2 Department of Mathematics,

FACUlty of ScIEnce AND ARTS,

Düzce University, Konuralp Campus,

DÜZCE-TURKEY

E-mail address: ${ }^{1}$ sarikayamz@gmail.com

E-mail address: ${ }^{2}$ yaldizhatice@gmail.com

${ }^{3}$ Department of Mathematics,

FACULTY OF SCIENCE,

BARTIN UNIVERSITY,

BARTIN-TURKEY

E-mail address: erdem1627@gmail.com 